\title{
Steuerung komplexer Systeme
}

Jürg Nadig

Jürg Nadig hat sich in früheren Beiträgen [1-3] kritisch mit den Wirtschaftlichkeitsverfahren von santésuisse auseinandergesetzt, dies unter besonderer Berücksichtigung seiner eigenen Fachrichtung, der medizinischen Onkologie. Im folgenden Beitrag skizziert er nun seine Vorstellung einer sinnvollen Steuerung des Gesundheitswesens: Die Monitorisierung der Behandlungsqualität nimm in seiner Konzeption eine zentrale Stellung ein.

Die Redaktion

1 Nadig J. Verdeckte Rationierung dank Wirtschaftlichkeitsverfahren? Schweiz Ärztezeitung. 2008; 89(20):855-60.

2 Nadig J. Replik auf die Stellungnahme von santésuisse. Schweiz Ärztezeitung. 2008;89(21):907-8.

3 Nadig J. Kommentar des Autors. Schweiz Ärztezeitung. 2008; 89(25):1122-3

Korrespondenz:

Dr. med. Jürg Nadig

Rebbergstrasse 7

CH-8157 Dielsdorf

juerg.nadig@hin.ch
Die zielgerichtete medikamentöse Tumorbehandlung geht Hand in Hand mit der zunehmenden Individualisierung in der Gesellschaft und nährt die Hoffnung, vermehrt auf den einzelnen Patienten zugeschnittene Behandlungen einsetzen zu können. Dieser individualisierten Therapie steht aber die politische Realität entgegen, die versucht, mit Managed Care, Fallpauschalen und bevorzugten Anbietern die Kosten zu kontrollieren, meint zu senken, um so das soziale Gesundheitswesen zu steuern.

Beiden Situationen gemeinsam ist der Versuch, komplexe Systeme zu steuern: einmal durch zunehmende Individualisierung der Behandlung, einmal durch zunehmende Pauschalisierung der Kosten. Damit ebenso widersprüchlich verknüpft sind steigende private Gewinne der pharmazeutischen Industrie, finanziert mit Geldern aus kollektiven Zwangssystemen, seien es Steuern und/oder die Kopfprämien der obligatorischen Krankenversicherung.

Der ökonomische Ansatz, das Gesundheitswesen allein über einen Preiswettbewerb zu steuern und gleichzeitig jedem Bürger einen fairen Zugang zum Gesundheitswesen zu erhalten, greift zu kurz. In den USA, dem Geburtsland des Managed Care, führte dieser Ansatz in den letzten dreissig Jahren zum teuersten, ineffizientesten und ungerechtesten Gesundheitswesen der Welt. Wurden die Preise einzelner Leistungen gesenkt, machte die Mengenausweitung den Verlust wieder wett. Statt den Behandlungsprozess zu optimieren, optimierten einzelne Akteure durch Kostenverschiebungen ihre Gewinne. Dies führte letztlich zu einem Nullsummenspiel mit sinkender Versorgungsqualität.

Langsam erkennen wir wieder, dass in der Medizin der Patient als Subjekt im Zentrum stehen muss und deshalb nur ein Wettbewerb zur Verbesserung des Patientennutzens ethisch vertretbar ist und die heutige Fehlentwicklung korrigieren kann. Nicht die Ökonomen sollen Ärzte und Pflegende, sondern Ärzte und Pflegende sollen den Behandlungsprozess im Interesse des Patienten lenken, damit die Vision einer Individualisierung der Behandlung überhaupt umgesetzt werden kann. Dazu braucht es aber nicht von den Krankenkassen ausgewählte Gatekeeper, die als politisch wiederbelebte Götter in Weiss die ganze Behandlung aus einer Hand wundersam steuern sollen, sondern es verlangt nach sich selbst regulierenden Behandlungsnetzwerken für einzelne Krankheiten mit einem Versorgungsauftrag, in denen die verschiedenen Fachleute gemeinsam versuchen, die ganze Behandlungskette zugunsten des Patientennutzens $z u$ verbessern. Ein solches Behandlungsnetzwerk für das Mammakarzinom zertifiziert dann nicht primär den einzelnen Eingriff oder das Spitalbett nach ISO-Normen, sondern ist verantwortlich für den grössten Patientennutzen in der ganzen Behandlungskette, von der Prävention über die Früherfassung bis zur Behandlung im fortgeschrittenen Stadium. Es koordiniert die Aufgaben der verschiedenen Akteure und optimiert die Schnittstellen. Gleichzeitig dienen solche integrierte Behandlungsnetzwerke als Aus-, Weiter- und Fortbildungsstrukturen, in denen Neuerungen in der Behandlung optimal umgesetzt werden können. Nur wenn in einer solchen Behandlungskette der Patientennutzen erfasst und offengelegt wird, kann der Patient sich für das Netzwerk mit dem besten Kosten-Nutzen-Verhältnis der Behandlung entscheiden.

Solche integrierten Netzwerke verlangen aber nicht nur eine Vollkostenrechnung, sondern vor allem auch eine Monitorisierung der Behandlungsqualität. Zu den Vollkosten fehlen den Kostenträgern in der Schweiz die Daten. Den Ärzten hingegen fehlen Instrumente zur Erfassung und Auswertung der Behandlungsqualität, so dass wir in der Schweiz beispielsweise die Veränderung der Versorgungsqualität als Folge der Einführung der Fallpauschalen (DRG) zur Finanzierung der Spitäler nicht werden überwachen können.

In einem Gesundheitswesen, in dem der Patientennutzen und nicht die niedrigen Krankenkassenprämien im Zentrum stehen, wird es die Aufgabe der betroffenen Fachgesellschaften sein, gemeinsam mit den andern Spezialisten Qualitätsindikatoren für ganze Behandlungsketten zu erarbeiten, um so die Behandlungsqualität zu verbessern, um längerfristig die Qualitätsdaten offenlegen zu können. Erst dann kann der mündige Patient seine individualisierte Behandlung mit der besten Qualität auswählen. Nur wenn es uns gelingt, sowohl die Weiterbildung als auch das Gesundheitswesens innovativ zum Wohl des Kranken zu steuern, lassen sich die neuen, teuren Behandlungsmöglichkeiten mittelfristig auch fair umsetzen. 